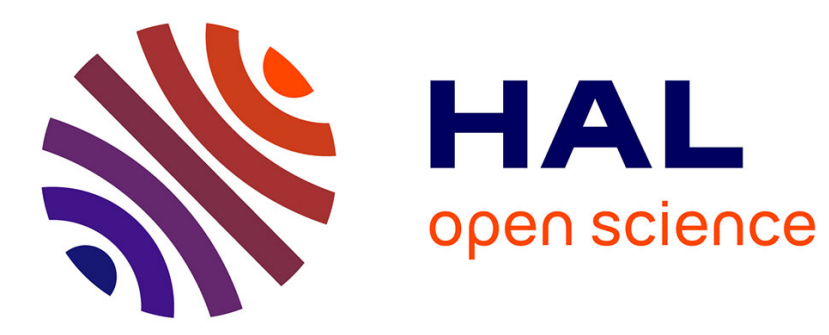

\title{
Self-sustained processes in the logarithmic layer of turbulent channel flows
}

Yongyun Hwang, Carlo Cossu

\section{To cite this version:}

Yongyun Hwang, Carlo Cossu. Self-sustained processes in the logarithmic layer of turbulent channel flows. Physics of Fluids, 2011, vol. 23, 10.1063/1.3599157 . hal-00913029

\section{HAL Id: hal-00913029 \\ https://hal.science/hal-00913029}

Submitted on 3 Dec 2013

HAL is a multi-disciplinary open access archive for the deposit and dissemination of scientific research documents, whether they are published or not. The documents may come from teaching and research institutions in France or abroad, or from public or private research centers.
L'archive ouverte pluridisciplinaire HAL, est destinée au dépôt et à la diffusion de documents scientifiques de niveau recherche, publiés ou non, émanant des établissements d'enseignement et de recherche français ou étrangers, des laboratoires publics ou privés. 


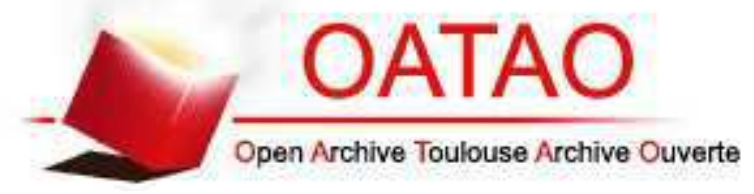

\section{Open Archive TOULOUSE Archive Ouverte (OATAO)}

OATAO is an open access repository that collects the work of Toulouse researchers and makes it freely available over the web where possible.

This is an author-deposited version published in : http://oatao.univ-toulouse.fr/ Eprints ID : 10378

To link to this article : doi:10.1063/1.3599157

URL : http://dx.doi.org/10.1063/1.3599157

To cite this version : Hwang, Yongyun and Cossu, Carlo Selfsustained processes in the logarithmic layer of turbulent channel flows. (2011) Physics of Fluids, vol. 23 ( $\mathrm{n}^{\circ}$ 6). ISSN 1070-6631

Any correspondance concerning this service should be sent to the repository administrator: staff-oatao@ listes-diff.inp-toulouse.fr 


\title{
Self-sustained processes in the logarithmic layer of turbulent channel flows
}

\author{
Yongyun Hwang ${ }^{1, a)}$ and Carlo Cossu ${ }^{2, b)}$ \\ ${ }^{1}$ LadHyX, CNRS-Ecole Polytechnique, 91128 Palaiseau, France \\ ${ }^{2}$ IMFT-CNRS, Allée du Professeur Camille Soula, 31400 Toulouse, France
}

\begin{abstract}
It has recently been shown that large-scale and very-large-scale motions can self-sustain in turbulent channel flows even in the absence of input from motions at smaller scales. Here we show that also motions at intermediate scales, mainly located in the logarithmic layer, survive when motions at smaller scales are artificially quenched. These elementary self-sustained motions involve the bursting and regeneration of sinuous streaks. This is a further indication that a full range of autonomous self-sustained processes exists in turbulent channel flows with scales ranging from those of the buffer layer streaks to those of the large scale motions in the outer layer.
\end{abstract}

The mechanisms by which turbulent kinetic energy is produced and transferred among different scales of motion is not yet completely understood in turbulent wall-bounded shear flows. A noteworthy feature of these flows is the ubiquity of streaky motions with typical spanwise scales ranging from $\lambda_{z}^{+} \approx 100$ in the buffer layer ${ }^{1,2}$ to $\lambda_{z} \approx 2 h$ for largescale motions in turbulent channel flows. ${ }^{3,4}$ Important progress has been realized in the early 1990s using the "minimal flow unit" approach where direct numerical simulations are performed in periodic domains of small streamwise $\left(L_{x}\right)$ and spanwise $\left(L_{z}\right)$ size, therefore avoiding motions at scales larger than the domain size. Using this approach it was shown ${ }^{5}$ that buffer layer streaks can self-sustain even when motions at larger scale are inhibited, and that their existence therefore relies on an autonomous process. The autonomous nature of the buffer layer cycle and the fact that at low or moderate Reynolds numbers most of the kinetic energy is produced in the buffer layer have promoted the view that the motions at large scales are formed from the aggregation of structures of smaller scale. ${ }^{6-9}$ In a recent study ${ }^{10}$ we have challenged this view by demonstrating that, in a turbulent channel flow, motions at the largest scales survive even when all motions at smaller scales are artificially quenched by using an artificially over-damped large eddy simulation. It was also shown that the self-sustained process at large scale $\left(\lambda_{z} \approx 1.5 h\right)$ bears many similarities with the one in the buffer layer in particular with the respect to the central role of the lift-up effect in the amplification of the streaks, ${ }^{11-13}$ their secondary instability ${ }^{14}$ and their breakdown. However, differently from the buffer layer process, the large scale selfsustained process includes the effect of the dissipation induced by smaller scales.

Recently the minimal flow unit approach, previously used to analyze the buffer layer structures, has been extended $^{15}$ to larger domains of spanwise size $L_{z}^{+}>\approx 300$. In these intermediate flow units the mean flow profile is real-

\footnotetext{
${ }^{a}$ Electronic mail: yongyun@ladhyx.polytechnique.fr.

b) Also at Département de Mécanique, École Polytechnique, 91128 Palaiseau, France. Electronic mail: carlo.cossu@imft.fr.
}

istic up to a given distance $y_{d}^{+} \approx 0.3 L_{z}^{+}$from the wall. ${ }^{15}$ Contrary to the minimal flow unit, intermediate flow units allow for self-sustained motions of scale smaller than the flow unit extension. The scope of the present investigation is to understand if in the intermediate flow units the motions at the scale of the flow unit $\left(L_{z}\right.$ and $\left.L_{x}\right)$ are self-sustained or if they are indirectly forced by self-sustained motions at smaller scales. To answer this question we apply the approach already used to isolate the self-sustained process at large scale ${ }^{10}$ to these intermediate flow units with $L_{z}^{+}$ranging from $\approx 400$ to $\approx 2000$. We anticipate that, at each selected scale, the largest allowed structures self-sustain without input from smaller scales, consistently with previous results ${ }^{16}$ that have shown that replacing the buffer layer with a model of rough wall has almost no influence on the motions sufficiently above the buffer layer.

As in Ref. 10, small scale active motions are gradually quenched using large eddy simulations (LES) with artificially increased damping. In LES the effect of small scale motions not resolved by the grid is taken into account by the residual stress tensor that is modeled with an eddy viscosity $\tau_{i j}-\tau_{k k} \delta_{i j} / 3=-2 \nu_{T} \bar{S}_{i j}$, where $\bar{S}_{i j}$ is the symmetric part of the resolved velocity gradient. We use the "static" Smagorinsky model $^{17}$ for the eddy viscosity $\nu_{T}=D\left(C_{S} \bar{\Delta}\right)^{2} \overline{\mathcal{S}}$, where $\overline{\mathcal{S}}=\left(2 \bar{S}_{i j} \bar{S}_{i j}\right)^{1 / 2}$ is a measure of the local shear and $D=1-$ $e^{-(y+/ A+) 3}$ is a damping function used to enforce physical behavior near the wall. The product $\left(C_{S} \bar{\Delta}\right)$ can be interpreted as the local mixing length, ${ }^{18}$ where $C_{S}$ is the Smagorinsky constant and $\bar{\Delta}$ is the LES filtering scale (related to grid size). The "static" Smagorinsky model is used because, unlike the better performing dynamic models, ${ }^{19}$ it inhibits any backward spectral energy transfer from the smaller to the larger scales, which is of crucial relevance in our approach. In principle, to remove small-scale active motions from the flow, one could increase the grid spacing. Following this approach, however, if all the intermediate scales are to be removed, the largest scale motions would be poorly resolved by the too coarse grid. We follow another approach: ${ }^{10}$ the grid is kept fixed at reasonable resolution and the effective mixing length is increased by increasing 
the Smagorinsky constant above the nominal value $C_{S}=0.05$ that provides the best a posteriori accuracy. ${ }^{20}$ The spatial scales and characteristics of the largest surviving motions found using this approach have been shown to be extremely similar to those of the "natural" large-scale motions. ${ }^{10}$ It was also verified, ${ }^{10}$ that those results were not sensitive to a change in the specific form of the artificial extra-dissipation.

The simulations have been performed with the diablo $\operatorname{code}^{21}$ based on the fractional-step method with semiimplicit integration in time and a mixed finite-difference and Fourier discretization in space. The solutions are assumed periodic in the streamwise and spanwise directions with fundamental wavelengths given by the size $L_{x}$ and $L_{z}$ of the simulated domains. Several domains have been considered and are listed in Table I. Constant mass flux is enforced during the simulations with constant Reynolds number $R e_{m}=U_{m} 2 h / \nu$ based on the bulk velocity $U_{m}$ $=\left(1 / 2 h L_{z}\right) \int_{-h}^{h} \int_{0}^{L_{z}} u d y d z$, the kinematic viscosity $\nu$, and the channel height $2 h$. The grid spacing is uniform in the streamwise and spanwise directions while grid-stretching is used in the wall normal direction. ${ }^{21}$ The grid spacing is kept constant for the different domain sizes with $\Delta_{x} / h=0.098$, $\Delta_{z} / h=0.047$, and $N_{y}=81$ points in the wall-normal direction for the cases at $R e_{m}=38133$ and $\Delta_{x} / h=0.049$, $\Delta_{z} / h=0.023, N_{y}=129$ at $R e_{m}=73333$. Typically, in the wall-normal direction the first grid point is below $y^{+} \approx 2$ and the maximum spacing is less than $\Delta_{y}^{+} \approx 70$ at the channel center.

A set of simulations at the reference value $C_{S}=0.05$ of the Smagorinsky constant is performed to validate the LES results against direct numerical simulation (DNS) at $R e_{m}=38133$. We have first verified (see Fig. 1) that in a relatively large box (Box0 with $L_{z}=1.5 h, L_{x}=3.14 h$ ) the mean flow profile and the velocity fluctuations (not shown) of our simulations agree with the ones ${ }^{22}$ computed by DNS in the same box at the same Reynolds number $\left(R e_{\tau}=957\right.$ in the LES and $R e_{\tau}=934$ in the DNS). Then, smaller computational domains (see Table I) with, respectively, $L_{z}^{+} \approx 900$ (Box1), 700 (Box2), and 400 (Box3), and $L_{x} / L_{z}=2.09$ are considered to exclude the largest motions while still including typical log-layer structures in the box in the spirit of Ref. 15. As reported in Fig. 1, in the intermediate flow units the mean flow remains accurate near the wall in a region extending up to $y_{d}^{+} \approx 0.3 L_{z}^{+}$(not shown) just as previously found in DNS. ${ }^{15}$ The largest structures found in the intermediate flow units consist in a single low-speed streak and a milder high speed streak both sinuously bent with a single wavelength in the domain (see panel $a$ in Fig. 2 for the Box1 flow unit), again in accordance with DNS results. ${ }^{15}$ These structures undergo sinuous oscillations interleaved with bursting epi-

TABLE I. Size of the different considered computational domains ("flow units").

\begin{tabular}{lccccc}
\hline \hline Case & Box0 & Box1 & Box2 & Box3 & Box4 \\
\hline$L_{z} / h$ & 1.50 & 1.00 & 0.75 & 0.50 & 0.38 \\
$L_{x} / h$ & 3.14 & 2.09 & 1.57 & 1.05 & 0.79 \\
\hline \hline
\end{tabular}

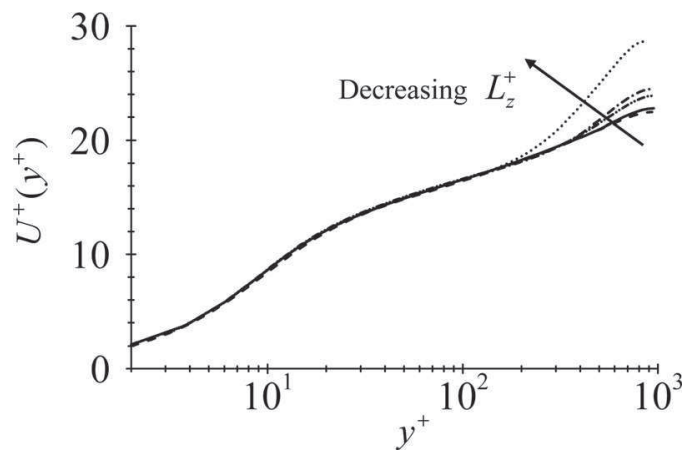

FIG. 1. Comparison of the mean flow profile $U^{+}\left(y^{+}\right)$of the reference LES simulation $\left(C_{S}=0.05, R e_{\tau}=957,-\right)$ in the large unit Box0 with the DNS data of del Álamo et al. (Ref. 22) $\left(R e_{\tau}=934,---\right)$ and with reference simulations (same $C_{S}=0.05$, same $R e_{m}$ ) in the smaller domains Box1 $\left(L z^{+}=693,--_{---}\right)$, Box2 $\left(L z^{+}=693,--_{--}\right)$, and Box3 $\left(L z^{+}=423,-\right.$ - -).
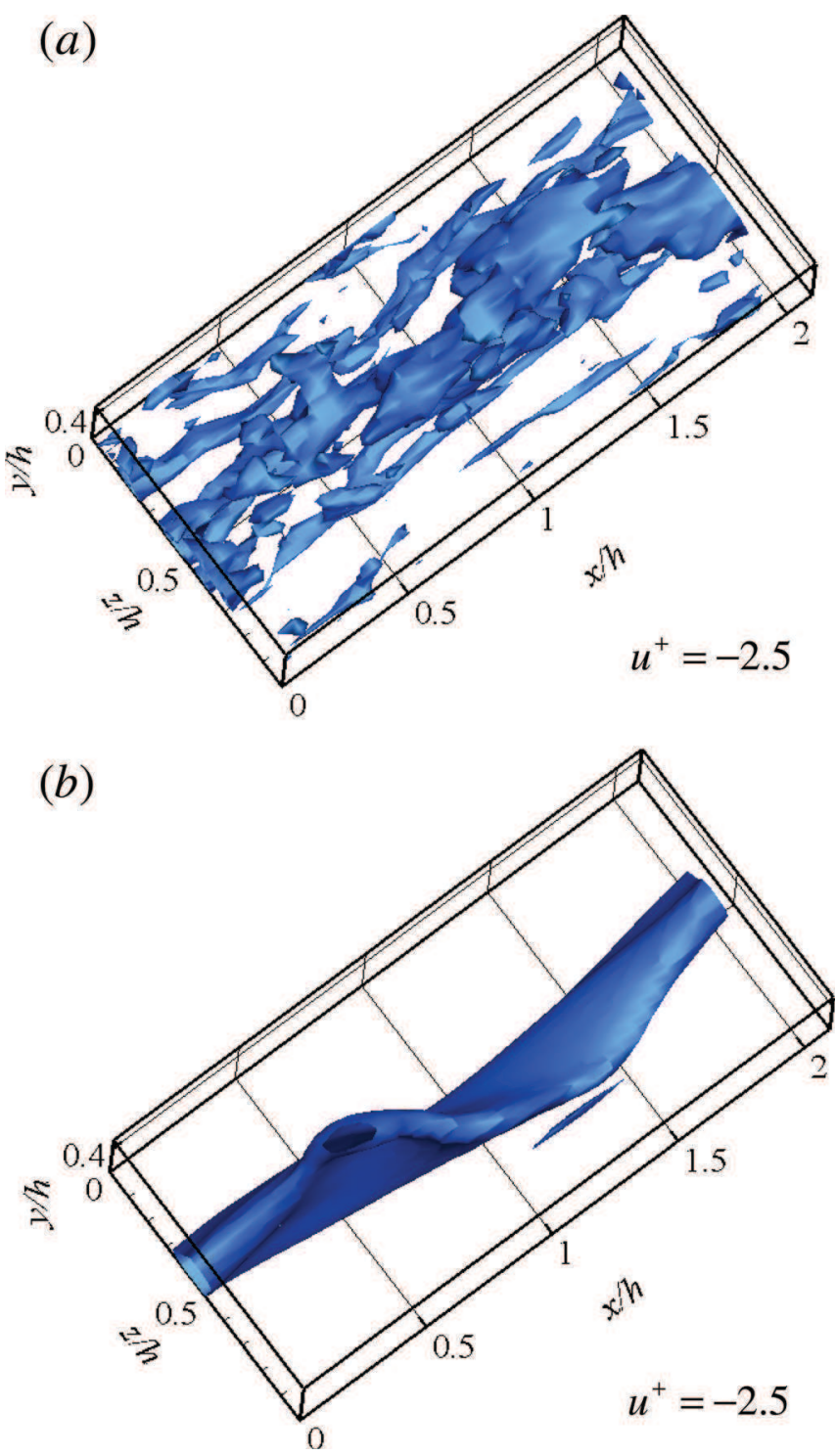

FIG. 2. (Color online) Instantaneous streamwise velocity fluctuation $\left(u^{+}=-2.5\right.$ iso-surface) in the Box1 flow unit for $R e_{m}=38133$ for, respectively, (a) the reference simulation $\left(C_{S}=0.05\right)$ and (b) the artificially overdamped simulation $\left(C_{S}=0.25\right)$. 
sodes. The usual peaks at $\lambda_{z}^{+} \approx 100$ and $\lambda_{x}^{+} \approx 1000$, corresponding to the buffer-layer self-sustained process, are found in the premultiplied spectra $k_{z} E_{u u}$ and $k_{x} E_{u u}$ of the streamwise velocity in all the considered flow units (see panels $a$ and $c$ in Fig. 3 for the Box 1 flow unit). Above $y^{+} \approx 50$ the most energetic scales correspond to the size of the box $\lambda_{z}^{+}=L_{z}^{+}$and $\lambda_{x}^{+}=L_{x}^{+}$.

To verify if the largest scale motions in the intermediate flow units are, or not, sustained by motions at smaller scales, the simulations are repeated using values of the Smagorinsky constant $C_{S}$ larger than the reference value. The Reynolds number is kept constant at $R e_{m}=38333$. The friction Reynolds number is not strongly affected by the increase in $C_{S}$; for instance, for the flow unit Box1, where $R e_{\tau}=934$ at the reference value $C_{S}=0.05$, it is found $R e_{\tau}=892$ for $C_{S}=0.1$ and $R e_{\tau}=1128$ for $C_{S}=0.25$. The smallest-scale structures are the first quenched when $C_{S}$ is increased above its reference value. The buffer-layer peaks $\lambda_{z}^{+} \approx 100, \lambda_{x}^{+} \approx 1000$ appearing in the premultiplied spectra of the streamwise velocity (see panels $a$ and $c$ of Fig. 3) have disappeared for $C_{S}=0.1$ in the Box1 flow unit (not shown). Motions at scales intermediate between the buffer layer peak and the box size are still active for $C_{S}=0.1$ but are quenched for $C_{S}=0.25$, with the only surviving motions being the ones with the flow unit size $\lambda_{z}^{+}=L_{z}^{+}=1128, \lambda_{x}^{+}=L_{x}^{+}=2362$, as reported in Figs. 3(b) and 3(d). The same phenomenology is observed in the premultiplied spectra of the spanwise and wall-normal velocity components. The structure of these surviving motions at the $L_{z}^{+}-L_{x}^{+}$scale, reported in Fig. 2(b), is similar to the one observed in the reference simulation (panel $a$ of Fig. 2), except for the absence of small scale structures. When $C_{S}$ is further increased, a critical value is attained where also these motions at $L_{z}^{+}-L_{x}^{+}$scale are quenched. As a general rule, the value of the critical $C_{S}$ increases with the dimension of the flow unit and tends to a constant for flow units larger than Box0. In the regime just below the critical $C_{S}$, the largest allowed scale motions are quenched also if the ratio $L_{x}^{+} / L_{z}^{+}$is reduced below $\approx 2$ at constant $C_{S}$. On the other hand, increasing the $L_{x}^{+} / L_{z}^{+}$ratio above $\approx 2.5$ leads to a richer dynamics with additional peaks at even larger scales. The ratio $L_{x}^{+} / L_{z}^{+}=2.09$, considered in the present study, therefore corresponds to the simplest dynamics at the chosen $L_{z}^{+}$scale. Essentially the same results are obtained at $R e_{m}=73333$.

A virtually identical scenario, except for a shift in the precise observed $C_{S}$ values, is observed for the other intermediate flow units at the same Reynolds number $R e_{m}=38133$, corresponding to $L_{z}^{+}=2021$ (Box0 at $\left.C_{s}=0.4\right), L_{z}^{+}=786\left(\right.$ Box2 at $\left.C_{s}=0.2\right)$, and $L_{z}^{+}=436$ (Box3 at at $\left.C_{s}=0.1\right)$, all with $L_{x}^{+}=2.09 L_{z}^{+}$. Just below the critical $C_{S}$ the surviving structures at the flow unit scale preserve an approximately similar structure across all the considered intermediate flow units in the region $y^{+}<\approx 0.3 L_{z}^{+}$where the mean flow profile remains accurate. Geometrical similarity (in the mean) of these largest scale surviving motions can be expected in the case of scales intermediate between the buffer layer and the outer ones $\left(L_{z}^{+}>\approx 400, L_{z} / h<\approx 1\right)$. Such a similarity would imply that the average characteristic lengths of the motion are proportional to $L_{z}^{+}$, which is the only characteristic length of the flow unit as the ratio $L_{x} / L_{z}$ is kept fixed. We verify if this is the case by analyzing how the $y_{\max }^{+}$wall-normal location of the peak in the root-meansquare profiles of the streamwise and wall-normal fluctuation velocity components $u_{r m s}\left(y^{+}\right), v_{r m s}\left(y^{+}\right)$. The results are reported in Fig. 4, where it can be seen that $y_{\max }^{+}$depends linearly on $L_{z}^{+}$except for the largest flow unit (Box0, not shown) for which $L_{z} / h>1$. The rms profiles are approximately geometrically similar (not shown) for $y^{+}<\approx 0.35 L_{z}^{+}$.
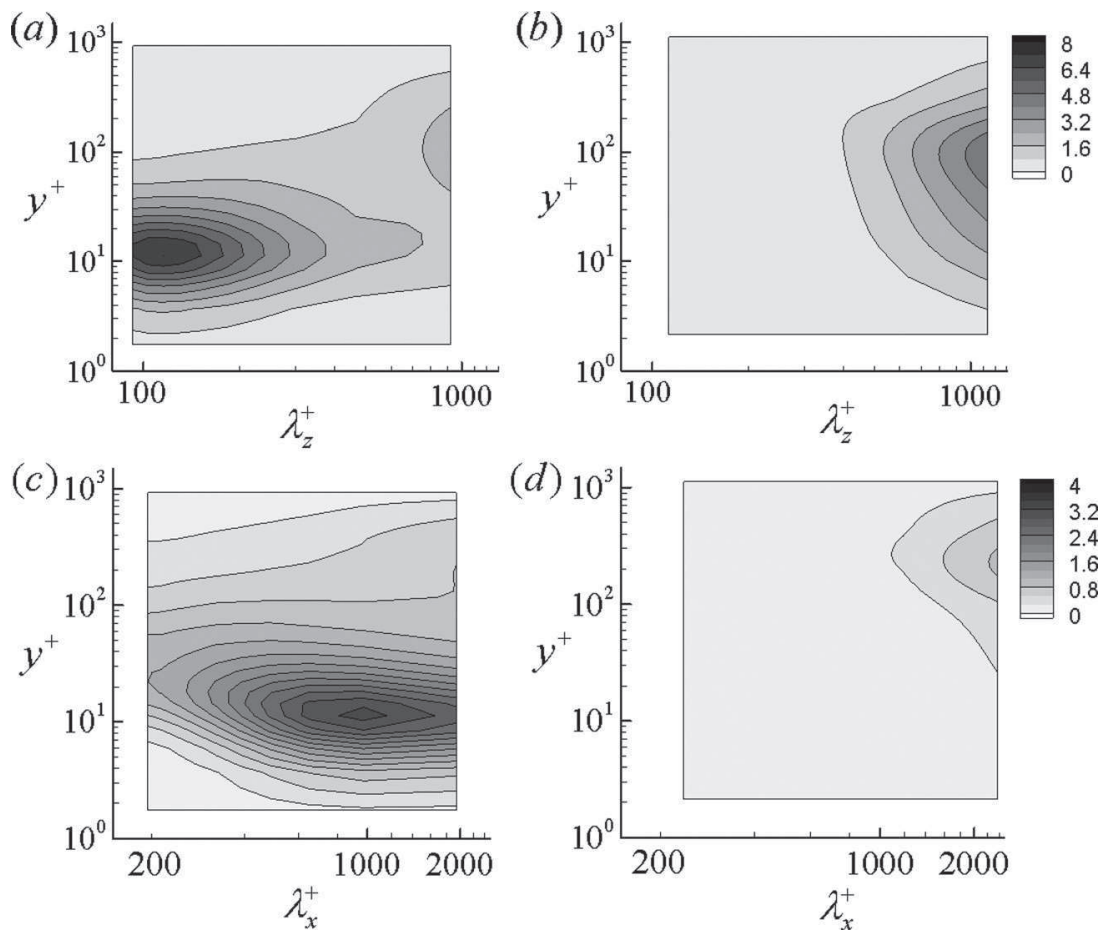

FIG. 3. Spanwise premultiplied power spectrum $k_{z} E_{u u}\left(\lambda_{z}\right)$ [(a) and (b)] and streamwise premultiplied power spectrum $k_{x} E_{u u}\left(\lambda_{x}\right)$ [(c) and (d)] computed in Box1 at $R e_{m}=38333$ for the same cases as in Fig. 2: (a) and (c) reference simulation with $C_{S}=0.05$; (b) and (d) over-damped simulation with $C_{S}=0.25$. 


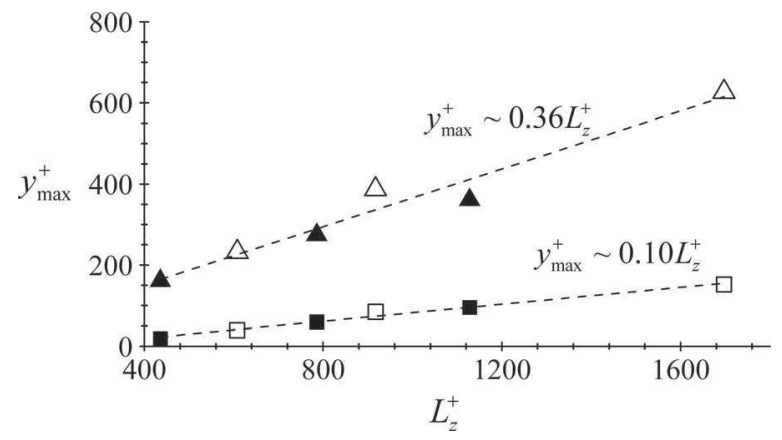

FIG. 4. Dependence on $L_{z}^{+}$of the peak $y_{\max }^{+}$of the $r m s$ profiles of the streamwise $\left[u_{r m s}\left(y^{+}\right)\right.$, squares $]$and wall-normal $\left[v_{r m s}\left(y^{+}\right)\right.$, triangles] fluctuation velocity components. The data correspond to the nearly-marginal overdamped simulations in the flow units listed in Table I for $\operatorname{Re}_{m}=38133$ (filled symbols, flow units Box1, Box2, Box3) and $R e_{m}=73333$ (empty symbols, Box2, Box3, Box4). The linear best fit to the data are also reported.

The wall-normal location of the peak in the spanwise and streamwise premultiplied spectra (panels $b$ and $d$ of Fig. 3 for the Box1 flow unit) also depends linearly on $L_{z}^{+}$.

We have also verified that a change in Reynolds number does not affect these results. Simulations at the higher $R e_{m}=73333$ have been performed in the flow units Box2 $\left(L_{z}^{+}=1697\right.$ for $\left.C_{s}=0.4\right)$, Box3 $\left(L_{z}^{+}=918\right.$ for $\left.C_{s}=0.3\right)$, and Box4 $\left(L_{z}^{+}=608\right.$ for $\left.C_{s}=0.2\right)$. For all these additional cases, all the $y_{\max }^{+}\left(L_{z}^{+}\right)$data points fall on the same linear fits found at the lower Reynolds number, as can be seen in Fig. 4 (empty symbols).

These results confirm and extend the ones recently obtained by Flores and Jiménez. ${ }^{15}$ We confirm that turbulence is self-sustained in a full range of intermediate flow units with size of the order of logarithmic layer structures. We have further demonstrated that the largest motions in these flow units, with scale equal to the flow unit size $L_{z}^{+}$and $L_{x}^{+}$, can sustain even in the absence of active motions of smaller scale where, however, the associated dissipation is still taken into account. A full range of single-scale elementary self-sustained active processes therefore exists with scales ranging from the one of the buffer layer cycle ${ }^{5}$ $\lambda_{z}^{+} \approx 100$ to the largest scales $^{10} \lambda_{z} \approx 1.5 h$. At least up to $y_{+} \approx 0.3 L_{z}^{+}$, these motions have, at first order, geometrically similar characteristic at intermediate scales which is compatible with the classical concept of attached eddies. ${ }^{23}$ The main mechanism by which these motions self-sustain is probably a coherent lift-up effect where coherent streamwise streaks are efficiently amplified from coherent streamwise vortices ${ }^{11-13}$ that are regenerated by the breakdown of the streaks. ${ }^{14}$ These self-sustained processes can probably be included as elementary fundamental bricks in a structural theory describing wall-bounded turbulent shear flows. Active research is currently pointing in this direction.

We gratefully acknowledge the use of the diablo code mainly developed by T. R. Bewley and J. Taylor.

${ }^{1}$ S. J. Kline, W. C. Reynolds, F. A. Schraub, and P. W. Runstadler, "The structure of turbulent boundary layers," J. Fluid Mech. 30, 741 (1967).

${ }^{2}$ J. R. Smith and S. P. Metzler, "The characteristics of low-speed streaks in the near-wall region of a turbulent boundary layer," J. Fluid Mech. 129, 27 (1983).

3J. Jiménez, "The largest scales of turbulent wall flows," Annual Research Briefs (Center for Turbulence Research, Stanford University, Stanford, CA, 1998).

${ }^{4}$ J. C. del Álamo and J. Jiménez, "Spectra of the very large anisotropic scales in turbulent channels," Phys. Fluids 15, L41 (2003).

5J. Jiménez and P. Moin, "The minimal flow unit in near-wall turbulence," J. Fluid Mech. 225, 213 (1991).

${ }^{6}$ A. E. Perry and M. S. Chong, "On the mechanism of turbulence," J. Fluid Mech. 119, 173 (1982).

${ }^{7}$ R. Adrian, S. Balachandar, and Z. Lin, "Spanwise growth of vortex structure in wall turbulence,” J. Mech. Sci. Tech. 15, 1741 (2001).

${ }^{8}$ C. D. Tomkins and R. J. Adrian, "Spanwise structure and scale growth in turbulent boundary layers," J. Fluid Mech. 490, 37 (2003).

${ }^{9}$ I. Marusic, "Unravelling turbulence near walls," J. Fluid Mech. 630, 1 (2009).

${ }^{10} \mathrm{Y}$. Hwang and C. Cossu, "Self-sustained process at large scales in turbulent channel flow," Phys. Rev. Lett. 105, 044505 (2010).

${ }^{11}$ J. C. del Álamo and J. Jiménez, "Linear energy amplification in turbulent channels,” J. Fluid Mech. 559, 205 (2006).

${ }^{12}$ G. Pujals, M. García-Villalba, C. Cossu, and S. Depardon, "A note on optimal transient growth in turbulent channel flows," Phys. Fluids 21, 015109 (2009).

${ }^{13}$ Y. Hwang and C. Cossu, "Linear non-normal energy amplification of harmonic and stochastic forcing in turbulent channel flow," J. Fluid Mech. 664, 51 (2010).

${ }^{14}$ J. Park, Y. Hwang, and C. Cossu, "On the stability of large-scale streaks in turbulent Couette and Poiseulle flows," C. R. Méc. 339, 1 (2011).

${ }^{15} \mathrm{O}$. Flores and J. Jiménez, "Hierarchy of minimal flow units in the logarithmic layer," Phys. Fluids 22, 071704 (2010).

${ }^{16}$ O. Flores, J. Jiménez, and J. del Álamo, "Vorticity organization in the outer layer of turbulent channels with disturbed walls," J. Fluid Mech. 591, 145 (2007).

${ }^{17}$ J. Smagorinsky, "General circulation experiments with the primitive equations: I. The basic equations," Mon. Weather Rev. 91, 99 (1963).

${ }^{18} \mathrm{P}$. Mason and N. Callen, "On the magnitude of the subgrid-scale eddy coefficient in large-eddy simulations of turbulent channel flow," J. Fluid Mech. 162, 439 (1986).

${ }^{19}$ For the further details on properties of the Smagorinsky model, the reader may refer, e.g., to Refs. 20, 24, and 25.

${ }^{20} \mathrm{C}$. Härtel and L. Kleiser, "Analysis and modelling of subgrid-scale motions in near-wall turbulence," J. Fluid Mech. 356, 327 (1998).

${ }^{21}$ T. Bewley, Numerical Renaissance: Simulation, Optimization and Control (Renaissance, San Diego, CA, 2008).

${ }^{22}$ J. C. del Álamo, J. Jiménez, P. Zandonade, and R. D. Moser, "Scaling of the energy spectra of turbulent channels," J. Fluid Mech. 500, 135 (2004).

${ }^{23}$ A. Townsend, The Structure of Turbulent Shear Flow, 2nd ed. (Cambridge Univertsity Press, Cambridge, UK, 1976).

${ }^{24} \mathrm{C}$. Meneveau and J. Katz, "Scale-invariance and turbulence models for large-eddy simulation," Annu. Rev. Fluid. Mech. 32, 1 (2000).

${ }^{25}$ P. Sagaut, Large Eddy Simulation for Incompressible Flows: An Introduction (Springer Verlag, Berlin \& Heidelberg, 2006). 